\title{
Adhesive-related warping of thin wooden bi-layers
}

\author{
Axel Rindler ${ }^{1,2} \odot \cdot$ Oliver Vay $^{1} \cdot$ Christian Hansmann ${ }^{1} \cdot$ Johannes Konnerth ${ }^{1,2}$
}

Received: 16 April 2019 / Published online: 12 September 2019

(c) The Author(s) 2019

\begin{abstract}
Warping of layered wood-based panels is still a challenging problem in the development of thin engineered wood products. Wood as an anisotropic and hydrophilic material tends to change its volume and mechanical properties with changing moisture content. Besides the wood components, also the mechanical properties of certain adhesives are sensitive to moisture changes. A moisture load onto the adhered wood is resulting in different stress and strain states between the adherends. It is expected that adhesives with different moisture-related properties participate differently to this interaction. To observe an adhesive-related warping, thin spruce/HDF (Picea abies and high-density fibreboard) bi-layers with identical material geometries were manufactured under laboratory conditions, using different wood adhesive systems, which are currently used in furniture and flooring industry [polyurethane (PUR), emulsion polymer isocyanate (EPI), polyvinyl acetate (PVAc), urea formaldehyde (UF) and ultra-low emitting formaldehyde amino adhesive (ULEF)]. The bi-layers were exposed to certain relative humidity conditions, and the resulting deformation was measured with a high-precision laser distance detector. Moisturedependent warping of the bi-layers was obtained in relation to the used adhesive systems. As a result of the study, it can be shown that initial warping after panel manufacturing strongly depends on the adhesive curing characteristics and, especially, on the amount of water that is released into the wood adherend. For the post-setting panel warping, a differentiation into two adhesive groups became visible: rigid and flexible adhesives. Rigid adhesives (UF and ULEF) showed a higher degree of warping compared to the group of flexible adhesives (PUR, EPI and PVAc).
\end{abstract}

Axel Rindler

a.rindler@wood-kplus.at

1 Wood K plus - Competence Centre for Wood Composites and Wood Chemistry, Altenberger Strasse 69, 4040 Linz, Austria

2 Department of Material Sciences and Process Engineering, Institute of Wood Technology and Renewable Materials, University of Natural Resources and Life Sciences (BOKU), Konrad Lorenz-Strasse 24, 3430 Tulln, Austria 


\section{Introduction}

Warping or distortion is a type of panel deformation out of its plane direction without the influence of an external mechanical load (Cai 2004; Cai and Dickens 2004; Suchsland and McNatt 1986). Despite some specific areas of application, i.e. bistable laminates (Etches et al. 2009), panel distortion is mostly unwanted and can be classified into the following distortion shape types: cup, bow, twist or saddle deformation (Brouse 1961; Gereke 2009).

Distortion is always generated by unbalanced stress and strain conditions that are caused by in-plane expansion or contraction of single panel constituents, based on the single material sensitivity to temperature, moisture or certain chemicals.

\section{Principle of wood panel warping}

In case of wood, a natural, anisotropic and hydrophilic polymeric material, swelling and shrinkage occur with changing moisture content (MC). Increasing moisture content results in swelling and occurs unless the fibre walls are fully saturated with water (fibre saturation point, FSP 30\%). In addition, thermal expansion and contraction are inherent to wood, but compared to moisture expansion with a comparable small magnitude (Bodig and Jayne 1982; Kollmann 1982).

The moisture-related swelling magnitude of wood depends on the species and significantly differs in longitudinal, radial and tangential directions, where a general relation between the directions of 1 to 10 to 20 (Kollmann 1982; Wagenführ and Scholz 2012) can be assumed.

The mechanical anisotropy of wood depends mainly on the local orientation of the wood fibres. The orientation of cellulose chains within them is responsible for the larger stiffness of wood in the longitudinal direction compared to the transversal direction (approx. 10:1). Further, the mechanical properties of wood, for example, elastic modulus $(E)$, are decreasing in all directions with increasing moisture content. Relevant for a bending application, the swelling increases the thickness of a layer and affects its second moment of area $(I)$ positively. Thus, in theory, an inverse relation between $E$ and $I$ is the result (Gerhards 2007).

The warping behaviour of a solid wood board is influenced by swelling and shrinking properties of wood in the longitudinal, tangential and radial directions, respectively, and the cell orientation, especially annual ring-orientation, in the board. Using solid wood boards, lamellas or veneers for panel assemblies results in a complex deformation process, where the intensity of distortion is quite sophisticated to foresee (Rindler et al. 2017).

Besides wood, the second main component in wood-based panel assemblies is the adhesive. The influence of different adhesive systems on the hygroscopic warping behaviour was observed on various wood-based panel assembly types.

The hygroscopic warping behaviour of 35-mm-thick 3-layered cross-laminated timber panels was investigated by Gereke (2009) by establishing a different moisture load between the two face layers for a duration of 31 days. Here, the influence of the stiffness 
of the used MUF and PUR resins was found to be low but present. He concluded that the stiffness of the adhesive plays a negligible role in the warping of the observed panels.

Hassani (2015) observed an adhesive-related difference in the warping behaviour of thin coaxial and cross-laminated 3-layered timber panels (Fagus sylvatica; middle lamella $d=4 \mathrm{~mm}$, face layers $f=d / 2$ ) manufactured with MUF, PRF and PUR. The panels were manufactured under humid climate $\left(20{ }^{\circ} \mathrm{C} / 95 \% \mathrm{RH}\right)$ and dried at $2 \%$ $\mathrm{RH}$ and again remoistened to $95 \% \mathrm{RH}$. Here, the aim was to primarily observe wood or bond line failure. For both layer orientations, the thin panels showed no cracking in the middle lamella but high warping signals. The MUF showed the highest warping, followed by PRF and PUR. Hassani (2015) concluded that the order of warping followed the same order of adhesive stiffness, where a higher stiffness of the adhesive generates higher warping.

Vailati et al. (2018) manufactured approx. 5-mm-thin bi-layers of spruce lamellas and beech veneer with different adhesive systems (PUR, PVAc, PRF, MUF, epoxy). In the first approach, the samples were exposed to $20{ }^{\circ} \mathrm{C} / 85 \% \mathrm{RH}$ until equilibrium followed by $20{ }^{\circ} \mathrm{C} / 35 \% \mathrm{RH}$ for $48 \mathrm{~h}$. In the second approach, a repetitive cycle of $20{ }^{\circ} \mathrm{C} / 15 \% \mathrm{RH}$ and $20{ }^{\circ} \mathrm{C} / 95 \% \mathrm{RH}$ for $12 \mathrm{~h}$ was applied. For both approaches, no statistically significant difference in warping could be observed, although for the first approach, a systematic difference between MUF and epoxy might be observable. Comparing the data of the two approaches, it becomes obvious that the adsorption and desorption phases of $12 \mathrm{~h}$ used in the second observation were too short to see differences in the signals. Thus, it is expected that a longer exposure to a specific RH is revealing stronger differences between the adhesive systems. Although the capability of absorbing water vapour (Wimmer et al. 2013) and the mechanical response to changing $\mathrm{RH}$ and temperature have been experimentally demonstrated for cured adhesive polymers (Jakes et al. 2015; Konnerth and Gindl 2008; Rindler et al. 2018; Stöckel et al. 2013), the effect of moisture sensitivity of adhesive on the warping behaviour of multi-layered panels remains unclear.

Moisture-driven swelling and shrinking of the wood-based adherends leads to considerable interfacial stress between the adhered components (Gindl et al. 2005) and directly affects the second main constituent in a wood-based bi-layer, namely the adhesive (Frihart 2009).

\section{Interaction of adhesive system and wood}

There are various adhesive systems applicable to solid wood bonding that can differ in their chemical structure and interaction with the adherend. Depending on the type of adhesive used, it is expected that the adhesive layer and/or the adherend is compensating occurring stresses and responds with either elastic, viscoelastic or nonreversible (plastic) deformation (Frihart 2009; Frihart and Wescott 2008).

The present investigation focuses on adhesives that exhibit different chemical and physical properties and are commonly used in flooring and furniture production, such as one-component polyurethane (PUR), two-component emulsion polymer isocyanate (EPI), polyvinyl acetate (PVAc), urea formaldehyde (UF) and ultra-low emitting formaldehyde amino adhesive (ULEF). 
PUR wood adhesives usually have a semi-crystalline thermoplastic character (Clau $\beta$ et al. 2011) and belong to the moisture curing adhesives. Simplified, PUR adhesives consist of soft and hard segments that interact by covalent bonds (crosslinking) and additionally by hydrogen bonds (Šebenik and Krajnc 2007). The soft segments derive from polyaddition of hydroxyl groups, forming urethane groups of less branched polyols and methylene diphenyl diisocyanate (MDI). The reactivity of the MDI isomers and moisture is responsible for the curing of the prepolymer after the adhesive application to the adherend. There is a huge variety of different precursors and additives (i.e. defoamers, fillers, etc.) for one-component PUR systems that allow adjusting the mechanical behaviour of the bond (Kläusler et al. 2013). PUR is very moisture resistant, and since it is not waterborne, no moisture transfer from the bond line is affecting the adherend due to gluing.

Similar to PUR, also EPI is a very moisture-resistant adhesive, curing via polyaddition and solvent displacement with characteristics of both, thermosetting and thermoplastic polymers. The system consists of a water-based emulsion, usually polyvinyl alcohol (PVA) that is derived by hydrolysis of polyvinyl acetate (PVAc) and is cured with an isocyanate cross-linker with one or more -NCO groups (Grøstad and Pedersen 2010). During gluing, different reactions and processes may take place, such as the water transport from the glue line to the wood adherend and reaction of $\mathrm{NCO}$ groups with water, other $\mathrm{NCO}$ groups, $\mathrm{OH}$ groups in PVA, functional groups and eventually to some degree with the hydroxyl groups of the wood adherend (Grøstad and Pedersen 2010; Qiao et al. 2000). The solid content usually is above $50 \%$ and varies depending on the implemented additives. Similar to PUR, also for EPI a couple of different additives are available to modify the later properties of the polymer and to increase the solid content (Grøstad and Pedersen 2010). The adhesive usually reaches its full mechanical strength and moisture resistance after 1-2 days of curing. Due to their moisture resistance, EPI and PUR act as moisture barriers. Mannes et al. (2012) detected a moisture concentration in wood close to the glue line for EPI and PUR, which could further affect the mechanical properties of the interphase zone.

Strongly related to the emulsion part of the EPI is PVAc, a thermoplastic adhesive curing via water loss. Without any additional chemical reaction, PVAc is very moisture sensitive which could lead to delamination when exposed to very humid climates. Usually, PVAc exhibits a solid content around 50\%, resulting in a tremendous water transfer through the adherend when hardening.

PVAc derives from a radical polymerization of vinyl acetate to high molecular PVAc chain molecules. Without crosslinking of the polymer chains, the cured adhesive is very sensitive to water and moisture (Dunky and Niemz 2013).

Amino adhesives such as UF and ULEF, usually have a solid content of $>60 \%$, are curing via polycondensation reaction and provide a high degree of crosslinking. The curing of UF is typically described to occur in two steps, the main reaction between urea and formaldehyde (alkaline methylation) and the final acid condensation reaction (Dunky and Niemz 2013). The reaction between urea and formaldehyde is reversible, thus UF is not stable to hydrolysis at high temperatures and very humid conditions, which can also negatively influence formaldehyde emissions. Since stronger regulations concerning formaldehyde emissions were established 
in the past years, amino adhesives such as ULEF are gaining more significance in production. A path to reduce the emission is to reduce the $F / U$ mole ratio, which also reduces the reactivity of the resin. An alternative is the addition of other reactants such as melamine or scavengers that react with the free formaldehyde (Henriques et al. 2017; Myers 1984). However, already very low melamine addition to UF leads to significantly better moisture resistance (Dunky and Niemz 2013). According to Frihart and Wescott (2008) and Frihart (2009), wood adhesives cannot only be classified chemically but also according to their interaction with wood. Highly cross-linked but rigid in situ polymerized adhesives, for example, amino resins, that are stabilizing the wood surface and enable a better strain distribution through the adherend are forming one group. The second group consists of slightly or not crosslinked pre-polymerized adhesives, for example, PUR, EPI or PVAc, that are more flexible and are able to distribute occurring strain through the adhesive (Frihart and Wescott 2008).

The present investigation is focused on identifying major differences in the warping intensity of the described adhesive systems. Therefore, two wood-based materials with different structural and mechanical characters were adhesively combined. A high-density fibreboard (HDF) with a homogenized fibre orientation, a moisture expansion coefficient (\% expansion per \% moisture content gain, CME) of approximately 0.04 in plane, 0.8 perpendicular to the plane direction and a modulus of elasticity (MOE) of around 4.3 GPa were used as the first layer. As second layer, an anisotropic spruce wood with a CME of approximately 0.19 in radial, 0.36 in tangential direction and a MOE of around $11 \mathrm{GPa}$ in longitudinal and $0.6 \mathrm{GPa}$ in transverse direction (Kollmann 1982; Niemz and Sonderegger 2017) was selected. With almost equivalent material thickness of HDF and spruce wood, spruce is the dominant deformation elicitor in this bi-layer, due to its higher CME in the transverse and radial directions.

For the present investigation, it is therefore hypothesized that in situ polymerized and pre-polymerized adhesive groups act differently when it comes to moisturedriven warping of thin wood-based bi-layers. Therefore, the applied moisture load is in a range of $\mathrm{RH}$ levels reflecting a realistic span of interior climate conditions occurring between winter and summer seasons (Rapp et al. 2011).

\section{Materials and methods}

\section{Sample preparation and conditioning}

Wood-based bi-layered panels were manufactured by using $1.4 \mathrm{~mm}( \pm 0.1 \mathrm{~mm})$ spruce veneer (Picea abies) from the same veneer batch with a density of $410 \pm 30 \mathrm{~kg} / \mathrm{m}^{3}$ on one side of the panel and a $1.6 \mathrm{~mm}( \pm 0.1 \mathrm{~mm})$ high-density fibreboard (HDF, Homadur B1, Homanit) derived from a dry process (amino resin binder) with a density of approx. $960 \pm 30 \mathrm{~kg} / \mathrm{m}^{3}$ on the other side. The spruce veneer originated from a slicing process and exhibited an annual ring interval varying between 1.6 and $2.6 \mathrm{~mm}$ along the veneer sheet width. The growth ring orientation was approx. $90^{\circ}$, applying for the whole veneer batch. The direction 
of the grain was oriented ideally parallel to the veneer sheet length. To obtain values independent of veneer side-related deformation, the adhesive application was conducted at the same veneer side always. The bi-layers were adhered with five different adhesive systems: a one-component polyurethane (1 K PUR; Loctite HB S 309 Purbond, Henkel AG, Sempach-Station Switzerland), an emulsion polymer isocyanate (EPI; Cascolit 4421nd hardener 2556, Akzo Nobel N.V., Amsterdam, Netherlands), a polyvinyl acetate (PVAc D3; Jowacoll 103.84, Jowat SE, Detmold, Germany), an urea formaldehyde (UF; Cascorit 1274 and hardener 7500, Akzo Nobel N.V., Amsterdam, Netherlands) and an ultra-low emitting formaldehyde amino resin (ULEF; LignuPro Zero A201 and hardener H203, Akzo Nobel N.V., Amsterdam, Netherlands).

Prior to pressing, the veneer and HDF panels were stored at $25{ }^{\circ} \mathrm{C}$ and $50 \%$ relative humidity $(\mathrm{RH})$ in a climate chamber for 7 days. For each individual assembly, the material was provided just before pressing in climatically isolated and sealed plastic bags to limit the change in moisture content (MC) of the material shortly before gluing. The adhesives were applied with a tooth spatula (A1 toothing size) to the HDF surface. To ensure an adhesive application quantity of approximately $140 \mathrm{~g} / \mathrm{m}^{2}$ wet for every panel, the application was performed at a laboratory balance. The pressing temperature and time were applied as suggested by the adhesive manufacturer. For the EPI and the PUR system, room temperature curing (approx. $20{ }^{\circ} \mathrm{C}$ ) and a pressing time of $420 \mathrm{~s}$ (EPI) and $20 \mathrm{~min}$ (PUR) were applied, respectively. For all other adhesives, the parameters were $90{ }^{\circ} \mathrm{C}$ and $60 \mathrm{~s}$. A pressure of $0.8 \mathrm{MPa}$ was used for all panels. In total eight panels with each of the five adhesives were manufactured. Since the used adhesives differ concerning their solid content (Table 1), the amount of water brought into the adherends and their resulting moisture expansion during the pressing are different for every adhesive type. Here, a comparably high temperature for PVAc was chosen to better support the water transfer through the adherend. To allow for full (post)curing of the panels, they were stacked and weighed down with a $10 \mathrm{~kg}$ weight at $25{ }^{\circ} \mathrm{C} / 50 \% \mathrm{RH}$ for 2 days to ensure sufficient curing of the adhesive and provide a similar initial distortion magnitude of the panels. The panels were cut to a size of $190 \times 150 \mathrm{~mm}^{2}$, hung on a grid with clamps, placed into a climate chamber with the longitudinal direction of the veneer oriented vertically to reduce influence of gravity on the panel deformation. The climate inside the chamber was changed sequentially after a period of 7 days, and deformation measurements were conducted. The order of established climate conditions, starting with the dry climates, was chosen to minimize the influence of a possible hydrolytic degradation of the adhesive.

Unjointed veneer and HDF specimen were used to determine the equilibrium moisture content of the panel materials separately according to EN 844-4. Therefore, the samples were stored in the same climate chamber. After each climate period, a minimum of three samples were removed and their mass was analysed before and after drying at $103{ }^{\circ} \mathrm{C}$ until constant weight. The order of established climate conditions, their duration and the resulting $\mathrm{MC}$ values are displayed in Fig. 1. 


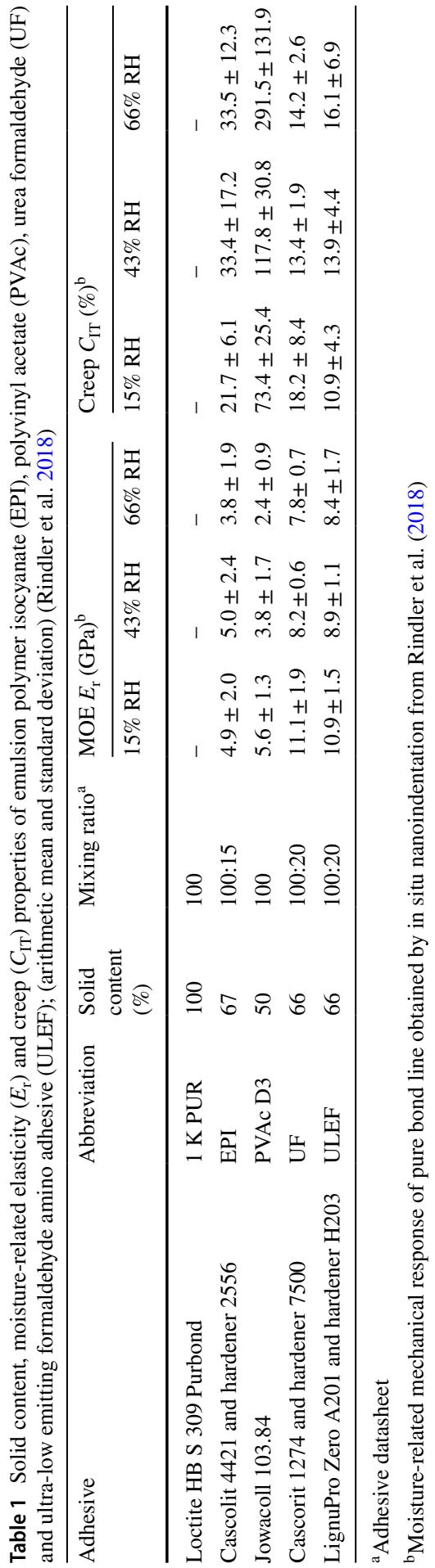




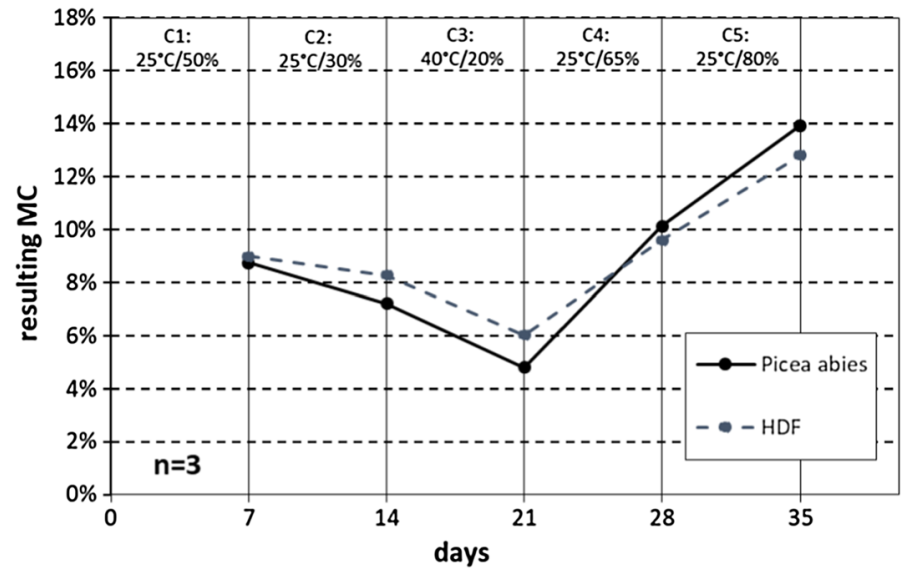

Fig. 1 Target equilibrium moisture content values according to the literature (Keylwerth and Noack 1964). Resulting equilibrium moisture content (MC) of Picea abies and HDF samples after storing under sequentially changing climate conditions $(\mathrm{C} 1-\mathrm{C} 5)$ for a duration of 7 days each

\section{Contactless deformation measurement}

A distortion-measuring table with a high-precision laser distance detector (optoNCDT ILD 1320-100, Micro-Epsilon Messtechnik GmbH, Ortenburg, Germany) was used to quantify the warping of the single panels after each climate step.

Therefore, the laser sensor was mounted to the centre of a $120-\mathrm{cm}$-long spirit level (laser joist), with the laser operation direction adjusted in a $90^{\circ}$ angle to the spirit level plane direction. A stable $590 \times 590 \times 350 \mathrm{~mm}^{3}$ steel frame was used, where the upper sections served as bearings for the spirit level. To obtain a quasiparallel in-plane direction between the spirit level and an ideal plane (coated $600 \times 180 \times 5.8 \mathrm{~mm}^{3}$ glass plate), the plate was positioned at height-adjustable screws with pointed tips (Fig. 2). The parallel adjustment of the glass plate was monitored via the laser distance detector to an accuracy of 1/100 $\mathrm{mm}$. As a result, the zero line is equivalent to the surface of the glass plate.

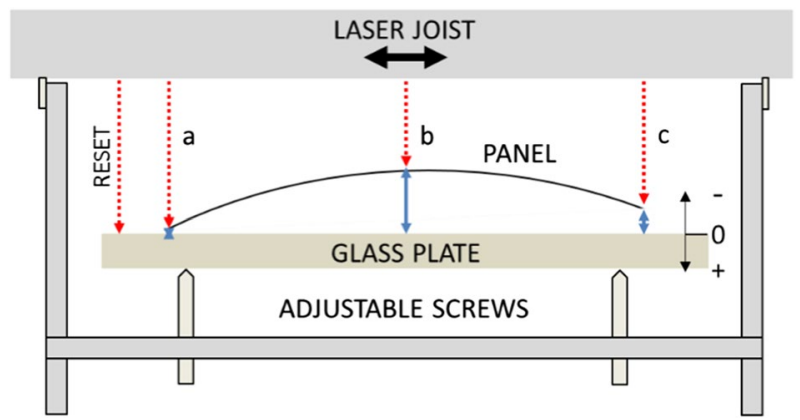

Fig. 2 Side view of the warping measuring table with dotted laser beams (dotted arrow) and the resulting difference between panel surface and glass plate surface (continuous arrows) 
For the panel measurement, a fixed position was marked on the glass plate, where the measured distance was reset to zero prior to every measurement.

For consistent measurements, all panels were marked at the same corner and centre positions as indicated in Fig. 3. After resetting of the laser beam, the panels were positioned on the glass plate with the HDF side upwards and the measurements were taken line-wise from left to right. The HDF side was chosen to be detected by the laser due to its comparably smooth surface.

With this pattern, the height of three longitudinal $\left(h_{1}\right)$ and three transverse bending curves $\left(h_{\mathrm{t}}\right)$ could be calculated by also considering the total panel thickness $\mathrm{t}_{\mathrm{p}}$ (Eq. 1) and averaged to a longitudinal $\left(h_{\mathrm{lm}}\right)$ and a transverse $\left(h_{\mathrm{tm}}\right)$ height of the bending curve for each panel.

$$
h_{\mathrm{t} i}=\left(\frac{(A-C)}{2}+B\right)+t_{p} \quad h_{\mathrm{l} i}=\left(\frac{(a-c)}{2}+b\right)+t_{p} \quad i=1,2,3
$$

Resulting positive values for either $h_{\mathrm{lm}}$ or $h_{\mathrm{tm}}$ represent concave, whereas negative values indicate convex panel deformation when HDF is on the upper side.

Due to an inappropriate determination of the accurate bowstring length of changing concave and convex panel deformation, instead of the average panel curvature the average transverse and longitudinal heights of the bending curves were used for indicating the panel warping.

\section{Micromechanical properties of the adhesives}

For the present study, moisture-related mechanical data from the same adhesives as used for the bi-layers were compared to the deformation behaviour of bi-layered panels. The stress brought to the adhesive layer due to bi-layer bending initiated by warping is assumed to be dominated by shear. Thus, knowledge of the shear modulus $(G)$ of the pure adhesive would be essential. Moisture-related in situ $G$ data of pure adhesive glue line are not available from the literature. However, a direct

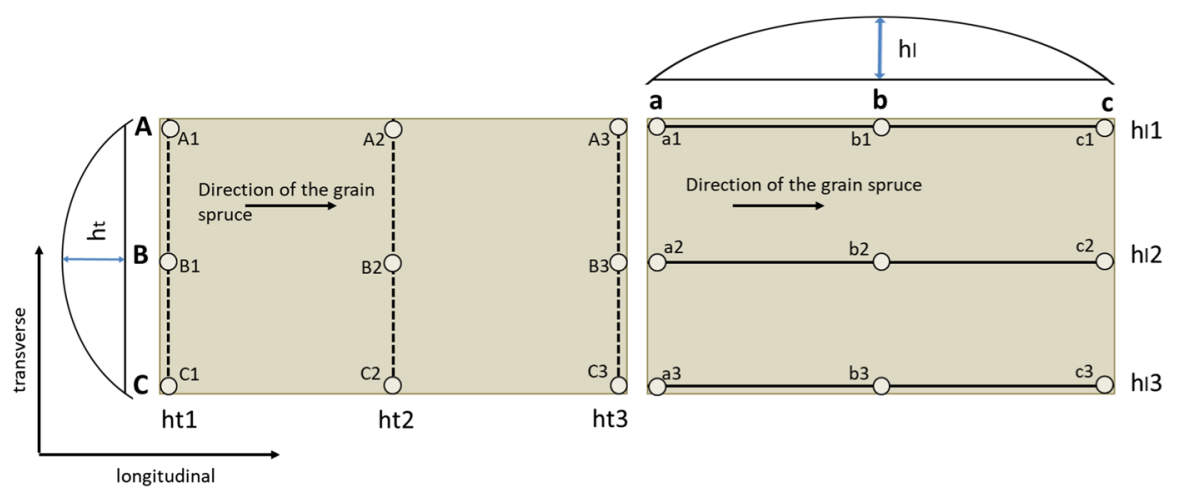

Fig. 3 Sketch of the measurement positions on the HDF panel surface resulting in different transverse (vertical lines, $\mathrm{ABC}$ ) and longitudinal (horizontal lines, abc) bending curves 
relation between $E$ and $G$ is typically applicable (Konnerth et al. 2006). For simplification, moisture-related in situ $E_{r}$ data were used directly from previous experiments (Stöckel et al. 2013; Rindler et al. 2018). For PUR, no directly comparable $C_{\text {IT }}$ values were available, that is why only $E_{\mathrm{r}}$ values were used with a value of $4 \mathrm{GPa}$ in accordance with values collected by Stöckel et al. (2013). The results for $E_{\mathrm{r}}$ and $C_{\mathrm{IT}}$ obtained from this experiment are listed as mean values in Table 1.

\section{Results and discussion}

In the following, the section "Results and discussion" is divided into two sections. In the section "Bi-layer deformation", the moisture-related transverse (Fig. 4) and longitudinal (Fig. 5) deformations are displayed in relation to the established MC for spruce wood. In the section "Adhesive mechanics on the observed bi-layer deformation", the slope values of Figs. 4 and 5 are plotted against reduced elastic modulus (Fig. 6) and indentation creep (Fig. 7) from Table 1.

\section{Bi-layer deformation}

The average height of transverse bending curves (htm) for the bi-layered panels manufactured with different adhesives is displayed in relation to the MC values achieved by Picea abies in the different climate steps in Fig. 4. Due to the different

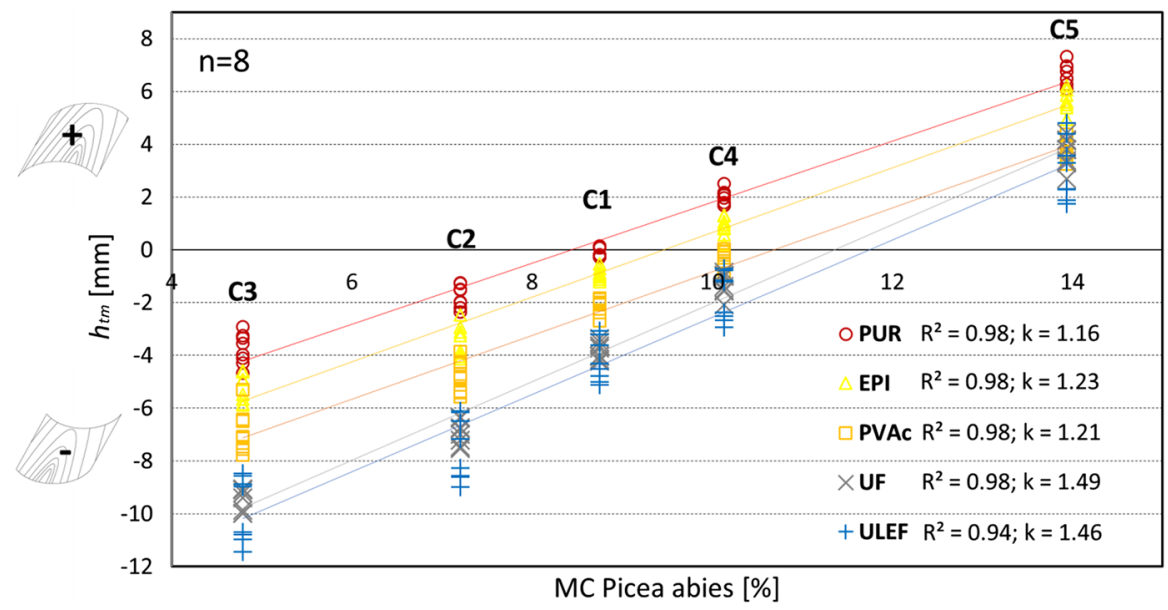

Fig. 4 Transverse deformation of spruce/HDF bi-layer versus moisture content (MC) of spruce. Convex $(+)$ and concave $(-)$ transverse deformations of the bi-layer with spruce veneer side up are schematically shown by the drawings on the left side. Data are displayed for bi-layers glued with either PUR (single component polyurethane), EPI (emulsion polymer isocyanate), PVAc (polyvinyl acetate), UF (urea formaldehyde) or ULEF (ultra-low emitting formaldehyde amino resin) stored in climate conditions C1-C5, where 1-5 indicate the chronology. Duration of storage was 7 days for each climate. Regression lines represent average values of transverse bending curve $\left(h_{\mathrm{tm}}\right)$ versus moisture content. $R^{2}$ is the coefficient of determination and $k$ the slope factor of the regression line 


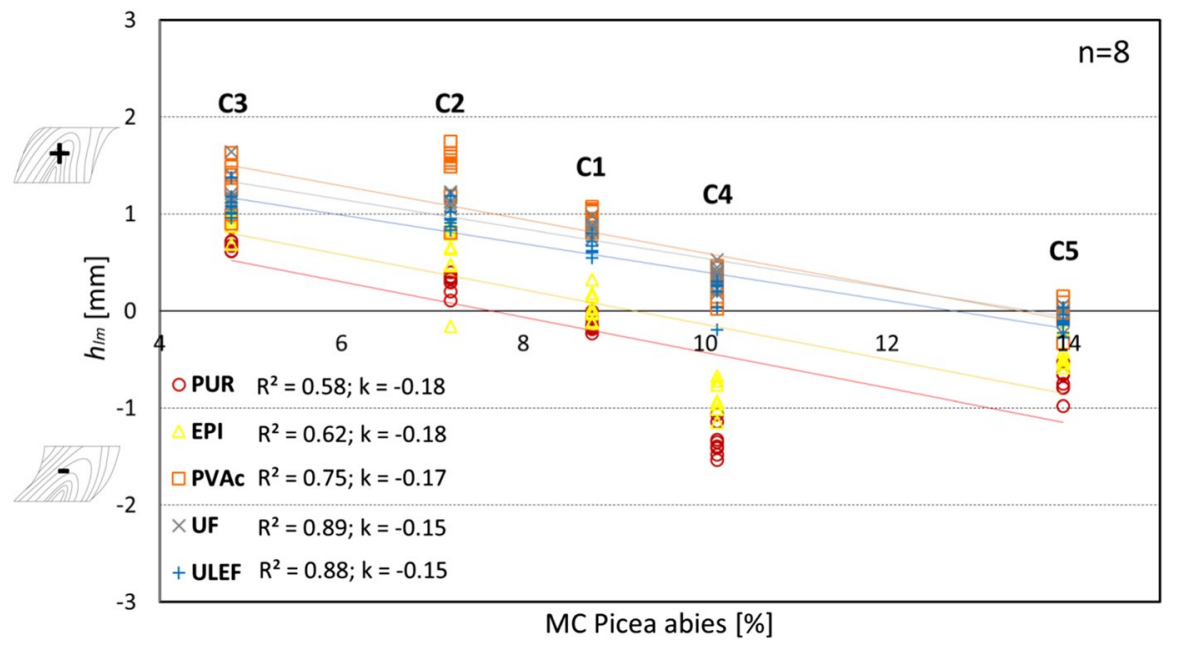

Fig. 5 Longitudinal deformation of spruce/HDF bi-layer vs. moisture content (MC) of spruce. Convex $(+)$ and concave (-) longitudinal deformations of the bi-layer with spruce veneer side up are schematically shown by the drawings on the left side. Data are displayed for bi-layers glued with either PUR (single component polyurethane), EPI (emulsion polymer isocyanate), PVAc (polyvinyl acetate), UF (urea formaldehyde) or ULEF (ultra-low emitting formaldehyde amino resin) stored at climate conditions C1C5, where 1-5 indicate the chronology. Duration of storage was 7 days for each climate. Regression lines represent average values of longitudinal bending curve $\left(h_{\mathrm{lm}}\right)$ versus moisture content. $R^{2}$ is the coefficient of determination and $k$ the slope factor of the regression line
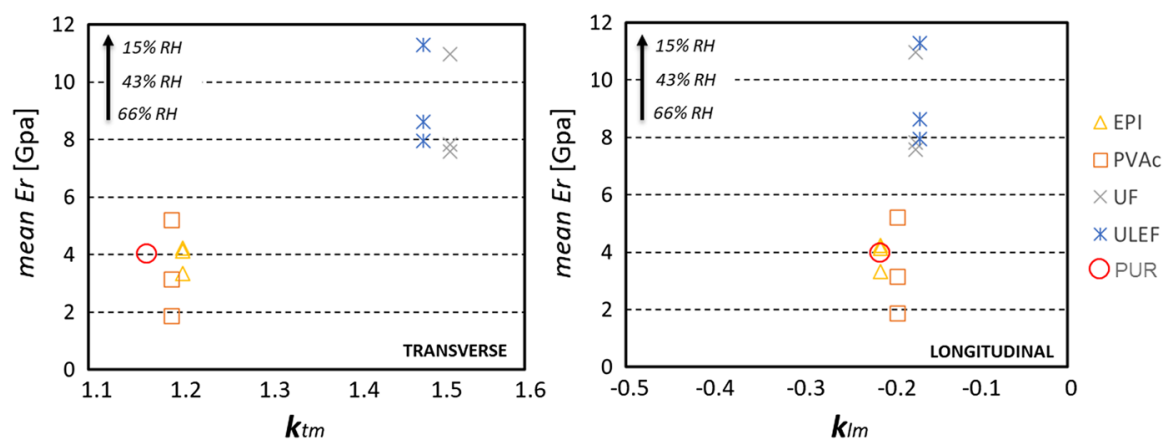

Fig. 6 Reduced elastic modulus $E r$ of adhesives versus slope factor $k$ of moisture-related warping of $\mathrm{HDF} /$ spruce bi-layers manufactured with the following adhesives: EPI (emulsion polymer isocyanate), PVAc (polyvinyl acetate), UF (urea formaldehyde), ULEF (ultra-low emitting formaldehyde amino resin) and PUR (polyurethane) from Stöckel et al. (2013). The $x$-axis displays the linear regression slope factor in transverse $\left(k_{\mathrm{tm}}\right)$ and longitudinal $\left(k_{\mathrm{lm}}\right)$ panel direction displayed in Figs. 4 and 5. On the y-axis, the mean moisture-related reduced elastic modulus $\left(E_{\mathrm{r}}\right)$ obtained from pure bond lines at $15 \%, 43 \%$ and $66 \%$ relative humidity RH of the same adhesives by means of nanoindentation (Rindler et al. 2018) is displayed. Higher $E_{\mathrm{r}}$ values of a certain adhesive correspond to lower RH as indicated by the arrow

adhesives, a systematic difference in the moisture content of the single samples at the specific climate steps cannot be excluded. However, a systematic difference in $\mathrm{MC}$ is related to a different adhesive to wood interaction of the chosen systems. 

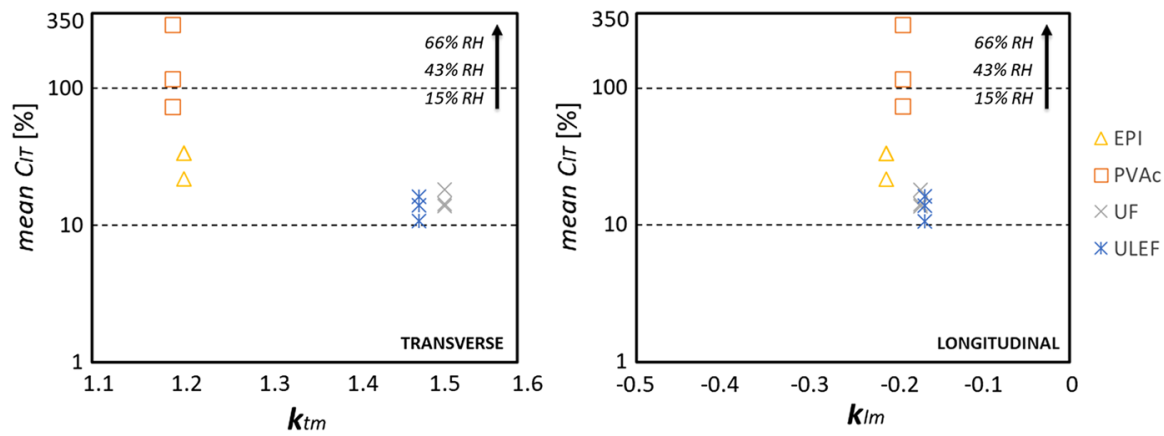

Fig. 7 Indentation creep $C_{\mathrm{IT}}$ of adhesives versus slope factor $k$ of moisture-related warping of HDF/ spruce bi-layers manufactured with the following adhesives: EPI (emulsion polymer isocyanate), PVAc (polyvinyl acetate), UF (urea formaldehyde) or ULEF (ultra-low emitting formaldehyde amino resin). The $\mathrm{x}$-axis displays the linear regression slope factor in transverse $\left(k_{\mathrm{tm}}\right)$ and longitudinal $\left(k_{\mathrm{lm}}\right)$ panel direction displayed in Figs. 4 and 5. On the y-axis, the mean moisture-related indentation creep $C_{\mathrm{IT}}$ obtained from pure bond lines at $15 \%, 43 \%$ and $66 \%$ relative humidity $\mathrm{RH}$ of the same adhesives by means of nanoindentation (Rindler et al. 2018) is displayed. Higher $C_{\mathrm{IT}}$ values of a certain adhesive correspond to higher $\mathrm{RH}$ as indicated by the arrow

All tested panels show both, negative and positive bending curve values indicating that concave as well as convex deformation was achieved depending on the moisture conditions for all adhesive systems in the transverse direction. Considering the panel width of $150 \mathrm{~mm}$, the gained values, ranging from approximately $-12 \mathrm{~mm}$ to $+7 \mathrm{~mm}$, are sufficiently high in magnitude to give proper insight into the warping behaviour of wooden bi-layers. All panel types independent of the adhesive type show deformation changes towards the same direction with changing MC. As the coefficient of moisture expansion (CME) of HDF (approx. 0.04\%/\%) is significantly smaller compared to Picea abies in transverse direction (approx. 0.19 radial and 0.36\% \% tangential) (Kollmann 1982; Niemz and Sonderegger 2017), the veneer layer mainly drives the deformation in transverse direction. Thus, the veneer swells and shrinks more dominantly compared to the HDF, resulting in a convex warping at dry climatic conditions and concave warping when there is a higher relative humidity.

Regarding the bending curve values of the panels using different adhesive types, it is to say that all groups show similar deviation of single values at the same MC values. In general, the deviation of all adhesives increases slightly for the very dry (higher scatter) and the very moist MC values compared to the more moderate moisture contents.

Focusing on climate $\mathrm{C} 1\left(8.8 \% \mathrm{MC}\right.$ at $25{ }^{\circ} \mathrm{C} / 50 \% \mathrm{RH}$ for Picea abies), it becomes visible that the PUR adhesive is the closest to the zero line, indicating a negligible transverse deformation after manufacturing. The other adhesives show negative values (concave warping) that increase in the following order: EPI, PVAc, UF and ULEF.

The panel glued with the PUR system showed less deformation after $\mathrm{C} 1$, as the adhesive requires no heat for curing and releases no water during the hardening (100\% solid content). Thus, no temperature or moisture load is brought to the veneer 
or the HDF during the pressing. As a result, there is no or only little change in MC in the adherends, and therefore, no moisture-driven swelling or shrinking occurs that leads to stress and an unbalanced strain distribution (warping) during the hardening of the wood-based bi-layer.

In addition, EPI does not require elevated temperature for curing but releases water during its hardening process (67\% solid content). The pressing time of EPI lasts for approximately $7 \mathrm{~min}$. During this time, the viscosity and strength of the dispersion part increase, while water enters the veneer and HDF layer. As a result, the wood substrate starts to expand while the curing of the adhesive proceeds. The adhesive reaches a high point of strength at the same time as the wood structure is swollen. Here, the adhesive fixes the adherends at higher moisture content under an expanded condition, different to spruce and HDF. After manufacturing, the wooden layers tend to equilibrate with the ambient atmosphere by starting to release water resulting in wood substrate shrinkage. If the shrinking coefficients of the layer materials show a disagreement, warping of the bi-layer is the result. In case of the EPI, the full curing procedure is usually finished after 2 days (Grøstad and Pedersen 2010), which enables the adherends to get rid of the water while the crosslinking of the adhesive might still be in progress. Thus, the appearance of a moisture load and bonding fixation of the adherends are temporally separated. This enables the adherend to acclimatize, reach equilibrium with the surrounding environment and swell or shrink in a less hindered way, resulting in less stress and consequently in less panel warping.

PVAc was manufactured at higher temperatures and is additionally releasing water during setting. The higher temperature is accelerating the diffusion of water, but it also increases the viscosity and shortens the hardening time of the adhesive straight after the temperature application, when the assembly is cooling. More water enters the wood layers in shorter time, while the viscosity increases, and therefore, the setting of the PVAc occurs faster. The fixation appears, while equilibrium of the adherends to surrounding climate is likely not established.

Both UF and ULEF require high temperatures and release water when curing. While for PVAc only water diffusion is necessary, UF and ULEF cure due to a polymerization reaction of their constituents. The polymerization occurs, while releasing moisture at generally higher temperatures. Usually, most of the final strength of the amino system is achieved directly after the pressing (Stöckel et al. 2010). Thus, it is expected that the fixation of the wood adherend relevant for warping appears at a MC state of the adherend far from equilibrium with the surrounding environment.

It is to conclude that this observed order of increasing deformation (PUR, EPI, PVAc, UF and ULEF) might be due to the solid content, the applied pressing temperatures and the rigid or flexible behaviour of the different adhesive systems. Compared to PUR, EPI and PVAc, the two amino resins (UF, ULEF) exhibit a more rigid character and are known to have higher stiffness.

For the drier climate conditions C2 and C3, some separation of the amino group and the other adhesives is visible. Coming to the humid climates $\mathrm{C} 4$ and $\mathrm{C} 5$, this separation disappears and UF and ULEF show similar values compared to PVAc in C5. 
The grouping becomes more obvious if one considers the regression lines for deformation response versus moisture content, with high coefficients of determination $\left(R^{2}\right)$, as indicated in Fig. 4. The slope factor $k$ of the regression line indicates the degree of change in moisture-related panel deformation. The higher the $k$-value, the more sensitive is the deformation response of the bi-layered panels to moisture. Thereby, it becomes visible that PUR, EPI and PVAc are performing similar with values of around 1.2, where UF and ULEF show a higher slope factor of around 1.5. Independent of the initial deformation after pressing, the higher slope factor is indicating a more sensitive deformation response to moisture changes in the UF and ULEF panels in transverse direction compared to the group of the other three adhesives.

The warping in longitudinal direction, as visible in Fig. 5, generally shows the opposite of the transverse values concerning moisture-related deformation direction. Here, the moisture-related swelling of the HDF (CME 0.04) is higher than the expansion of spruce in longitudinal direction (CME approx. 0.02). This results in a convex deformation with decreasing and a concave deformation with increasing relative humidity. However, with a range of $+2 \mathrm{~mm}$ to approximately $-1 \mathrm{~mm}$ in relation to a panel length of $190 \mathrm{~mm}$, the longitudinal deformation occurs with an order of magnitude lower as in the transverse direction.

In general, the longitudinal average bending curve values of all adhesives show a comparable deviation at all climate steps.

For climate C1, again PUR and EPI show the closest values to zero and therefore the smallest initial deformation. Interestingly UF and ULEF show comparable deformation to PVAc, which provides the values farthest from the zero line. This picture is mostly equal to the other climate steps C2-C5.

Implementing a regression line for each adhesive type with the coefficients of determination $\left(R^{2}\right)$ as indicated in Fig. 5, it becomes obvious that only the amino resins are sufficiently predicted with a linear behaviour. The comparably lower $\mathrm{R}^{2}$ is based on the more pronounced deviation from linearity as visible in C2 for PVAc and in C4 for PUR and EPI. In C2, the PVAc shows the strongest deformation compared to all climates. This is interesting, since the following climate step C3 aims for an even lower MC but shows a comparably smaller distance to the zero line. A similar picture is visible for PUR and EPI with a strong convex deformation in C4 that seems to disappear and decrease for an even higher $\mathrm{MC}$ in $\mathrm{C} 5$. Here, the large climatic gap between $\mathrm{C} 3\left(40^{\circ} \mathrm{C} / 20 \% \mathrm{RH}\right)$ and $\mathrm{C} 4\left(15^{\circ} \mathrm{C} / 65 \% \mathrm{RH}\right)$ has to be considered. After 7 days in climate $\mathrm{C} 4$, the gap to $\mathrm{C} 3$ was too large to reach the target MC. Therefore, the swelling of the wood structure was not finished, and stress was still built up in the joint. Interestingly, this gap seems to have no influence on the transverse direction for the panels bonded with PUR and EPI, but only on the longitudinal panel direction. In longitudinal panel direction, the CME of spruce is lower, but its stiffness is higher. Higher stiffness means a low hindered strain can result in high stress. PVAc, UF and ULEF are mechanically sensitive to moisture changes, decrease their elastic modulus and increase their creep behaviour, whereas PUR and EPI do not respond to moisture to the same extent (Rindler et al. 2018; Stöckel et al. 2013). In fact, the moisture-sensitive adhesives show very similar values after being exposed to the $\mathrm{C} 4$ climate for 7 days. Although the amino resins exhibit a higher 
stiffness compared to PUR and EPI, they appear to be less sensitive to this large moisture gap between $\mathrm{C} 3$ and $\mathrm{C} 4$. Further, PVAc is in a stiffness range comparable to PUR and EPI (approx. $5 \mathrm{GPa}$ at $15 \% \mathrm{RH}$ ), but the panel shows a similar warping behaviour to UF and ULEF. This is an indicator that besides the magnitude of stiffness, also the moisture sensitivity of the adhesive could be of more importance here. However, between C4 and C5, the climatic gap was less intense, and the 7 days seemed to be sufficient to come closer to the target MC, as the swelling stress is assumed to decrease, and the system started to relax. Hence, the deformation values of PUR and EPI in C5 are closer to the zero line compared to C4.

Despite the low $R^{2}$ values, the slope factor $k$ of linear regression was used to compare the trends of the moisture-dependent deformation intensity of the panels. A distinct differentiation between the adhesives is not possible, as the values are very small due to the low signal intensity of the longitudinal deformation. Compared to the transverse direction, the influence of the adhesive on the longitudinal deformation seems to be less pronounced.

\section{Influence of adhesive mechanics on bi-layer deformation}

The $k$ values of the linear regression of the average bending curves and the established MC (Figs. 4, 5) were plotted against mechanical data of the adhesives obtained from nanoindentation. The mean values of the reduced elastic modulus $E_{\mathrm{r}}$ were measured at $15 \%, 43 \%$ and $66 \%$ RH for EPI, PVAc, UF and ULEF (Table 1) and are opposed to the $k$ values for the bi-layered panels with the same adhesives in Fig. 6.

The upper symbol of each adhesive represents the average $E_{\mathrm{r}}$ value at $15 \% \mathrm{RH}$, while the lowest $E_{\mathrm{r}}$ value corresponds to the highest $66 \% \mathrm{RH}$.

From the diagram, it seems to be evident that the mechanical difference of the used adhesive systems plays a relevant role in the warping behaviour of the tested bi-layer. Further, it is expected that the adhesives` mechanical sensitivity to moisture could play a relevant role too, which is not revealed by the shown figures. In general, it is to say that pure EPI and PVAc bond lines perform with $E_{\mathrm{r}}$ mean values clearly below $6 \mathrm{GPa}$, were bond lines of UF and ULEF reveal higher $E_{\mathrm{r}}$ values starting from around $8 \mathrm{GPa}$. EPI with an $E_{\mathrm{r}}$ variation range of approximately $1 \mathrm{GPa}$ between $15 \%$ and $66 \% \mathrm{RH}$ is the most stable adhesive when exposed to moisture, whereas PVAc, UF and ULEF change their modulus in a variation range of approximately $3.5 \mathrm{GPa}$ between $15 \%$ and $66 \%$ RH. More detailed findings on the moisture-related reduced elastic modulus of these adhesives are described elsewhere (Rindler et al. 2018).

In the transverse direction (left diagram of Fig. 6), besides a slope factor $k$ related to grouping of adhesives, also a grouping related to the mechanical properties of the adhesives is visible. EPI and PVAc form a group with considerably lower $E_{\mathrm{r}}$ and lower $k$ values compared to UF and ULEF that perform nearly identical. For transverse panel direction, it seems that adhesives with higher $E_{\mathrm{r}}$ tend to show slightly higher moisture-related deformation response $(k)$ than adhesives with a lower $E_{\mathrm{r}}$ value. 
The slight differences visible between the adhesives for the longitudinal direction (right diagram of Fig. 6) are rather negligible; additionally, the coefficient of determination is quite weak. In this case, the different $E_{\mathrm{r}}$ values of the adhesives do not seem to affect the moisture-related deformation intensity of the longitudinal panel direction.

In Fig. 7, the achieved $k$ values of the linear regression between average bending curves and established MC (Figs. 4, 5) are plotted against moisture-related average creep values of the adhesives obtained from nanoindentation. Indentation creep $\left(C_{\mathrm{IT}}\right)$ was measured in situ in the adhesive bond line at $15 \%, 43 \%$ and $66 \% \mathrm{RH}$ for EPI, PVAc, UF and ULEF (Table 1). With respect to the $C_{\mathrm{IT}}$ values of the adhesives, it is to say that all adhesives show at least slight changes in their creep behaviour with changing RH. PVAc reveals by far the highest creep and the strongest sensitivity to moisture compared to EPI, UF and ULEF. The remaining adhesives respond quite stably with changing MC, whereas EPI in general shows slightly higher values compared to UF and ULEF, which perform quite similar. A more detailed description and discussion of the indentation creep findings were conducted elsewhere (Rindler et al. 2018).

Again, for the transverse panel direction (left diagram of Fig. 7), a grouping is visible with EPI and PVAc showing higher $C_{\mathrm{IT}}$ and lower $k$ values compared to UF and ULEF with lower $C_{\mathrm{IT}}$ and higher $k$ values. This could be seen as an indicator that bi-layers using adhesives with a higher affiliation to creep tend to show less warping compared to panels manufactured with less creeping adhesives. Similar to Fig. 6, no relation between $C_{\mathrm{IT}}$ and $k$ could be observed for longitudinal direction (right diagram of Fig. 7).

As creep is a long-term behaviour, probably some relaxation of the bi-layer should be observable when storing it for a longer period of time in a similar ambient. However, a related type of experiment was not performed within the frame of this study.

Two different types of adhesive-related warping were identified in the present study: initial warping immediately after pressing and post-setting warping of the final panel.

Initial warping is strongly related to the pressing temperature applied as well as the water balance of the adhesive. The water content of the applied adhesive together with the capability of the adhesive to release (polycondensation adhesives) or consume (PUR) additional water during the curing is assumed to influence the moisture content of the wood adherend. Although the described parameters seem to be common knowledge in wood science, the literature sufficiently describing their interaction and effect on initial panel warping is very limited.

As PUR does not release but requires a certain amount of moisture for hardening, it was found to be the most stable adhesive concerning initial warping, as it did not force an intense change in MC in the wood. In a quite different but still related context, PUR was used to reduce the distortion of green-glued glulam beams (Eriksson et al. 2004).

In contrast to several observations in the literature where a negligible influence of the adhesive to the hygroscopic warping behaviour of 2- and 3-layered wood panels was concluded (Gereke 2009; Vailati et al. 2018), the present study reveals clear 
effects of the adhesive system on thin wooden bi-layer post-setting warping. However, the present findings are in line with other empirical investigations reported in the literature (Blanchet et al. 2003; Hassani 2015; Tobisch 2006), which also state that differences in the warping behaviour between different wood adhesives are given and should not be neglected. Concerning the moisture-related adhesive mechanics, Blanchet et al. (2003) identified a grouping between amino resins and PUR and PVAc, similar to the results reported in the present study. Additionally, in fundamental wood adhesive literature (Frihart 2009; Frihart and Wescott 2008), a possible influence of adhesive properties on the dimensional stability has been proposed. Similar to the present study, Hassani (2015) and also Tobisch (2006) found a stronger warping for 3-layered panels manufactured with a typically stiffer MUF adhesive compared to panels produced with more flexible PUR and PVAc adhesives.

The adhesive in a bi-layer is located in the core of the panel and, thus, is not subjected to bending loads. Shear is the load type that stresses the bond line in such an assembly, thus the shear modulus of the adhesive would have more relevance for the assembly than the elastic modulus.

The findings in the present paper are based on observations on thin bi-layers with a comparably large adhesive amount in relation to the total assembly (1/30 assuming $0.1 \mathrm{~mm}$ adhesive layer thickness). With the help of this specific specimen design, it was possible to detect a relevant amount of warping. It is assumed that the observed magnitude of adhesive-related warping is expected to be clearly lower for bi-layers with thicker adherend material or multi-layered panels with a symmetrical layer assembly.

For the tested panels, the difference in the observed adhesive-related warping is present. In particular when it comes to very thin assemblies, the moisture-related properties of the adhesive and its interaction with the adherend should not be neglected but considered in the design.

\section{Conclusion}

The effect of the adhesive system on the moisture-related warping could be demonstrated for thin spruce/HDF bi-layers. The used measuring devices and chosen sample treatment provided sufficient and reliable deformation results. Despite identical assemblies, differences in panel deformation could be observed for the tested bi-layers that might be related to the different adhesive systems used. In general, two different degrees of moisture-dependent panel warping were observed, especially in the transverse panel direction, and could be defined as initial warping after pressing and post-setting warping. Initial warping after panel manufacturing strongly depends on the adhesive curing/setting characteristics, its requirement of a higher temperature during the hardening and, especially, on the amount of water that is released into the wood adherend. Here, clear differences could be observed between the tested adhesives. Furthermore, a differentiation into rigid and flexible adhesives became visible for the post-setting panel warping. Rather flexible adhesives that tend to comparably higher creeping (PUR, EPI and PVAc) also showed a lower degree of warping 
compared to stiff and rigid adhesives such as UF and ULEF. In the longitudinal panel direction, no influence of the adhesive group on the deformation intensity was observed, since the deformation signal was too weak to reliably distinguish between adhesive groups.

Acknowledgements Open access funding provided by University of Natural Resources and Life Sciences Vienna (BOKU). The authors are thankful for financial support for the project "Wood: next generation materials and processes-from fundamentals to implementations", funded by the Austrian Research Promotion Agency (FFG) (Project No. 844.608).

Open Access This article is distributed under the terms of the Creative Commons Attribution 4.0 International License (http://creativecommons.org/licenses/by/4.0/), which permits unrestricted use, distribution, and reproduction in any medium, provided you give appropriate credit to the original author(s) and the source, provide a link to the Creative Commons license, and indicate if changes were made.

\section{References}

Blanchet P, Beauregard R, Erb A, Lefebvre M (2003) Comparative study of four adhesives used as binder in engineered wood parquet flooring. For Prod J 53:89

Bodig J, Jayne BA (1982) Mechanics of wood and wood composites. Van Nostrand Reinhold Company Inc., New York

Brouse D (1961) Some causes of warping in plywood and veneered products Forest Products Laboratory. US Department of Agriculture, Madison

Cai Z (2004) Evaluating the warping of laminated particleboard panels. In: 7th Pacific rim bio-based composites symposium, vol 2, pp 69-79

Cai Z, Dickens JR (2004) Wood composite warping: modeling and simulation wood and timber. Science 36(2):174-185

Clauß S, Dijkstra DJ, Gabriel J, Kläusler O, Matner M, Meckel W, Niemz P (2011) Influence of the chemical structure of PUR prepolymers on thermal stability. Int J Adhes Adhes 31:513-523

Dunky M, Niemz P (2013) Wood-based panels and adhesives: technology and influencing factors. Springer, Berlin. https://doi.org/10.1007/978-3-642-55938-9

Eriksson J, Ormarsson S, Petersson H (2004) An experimental study of shape stability in glued boards. Holz Roh-Werkst 62:225-232. https://doi.org/10.1007/s00107-004-0468-z

Etches J, Potter KD, Weaver P, Bond I (2009) Environmental effects on thermally induced multistability in unsymmetric composite laminates. Compos A Appl Sci Manuf 40:1240-1247. https://doi. org/10.1016/j.compositesa.2009.05.018

Frihart CR (2009) Adhesive groups and how they relate to the durability of bonded wood. J Adhes Sci Technol 23:601-617. https://doi.org/10.1163/156856108X379137

Frihart CR, Wescott JM (2008) Why do some wood-adhesive bonds respond poorly to accelerated moisture-resistant tests? In: 9th Pacific Rim bio-based composites symposium: innovation and challenges in bio-based composites-how far to the new frontier? 5th-8th November 2008, Rotorua, New Zealand.[S1: sn], pp 51-58

Gereke T (2009) Moisture-induced stresses in cross-laminated wood panels Dissertation Eidgenössische Technische Hochschule ETH-Zürich No. 18427

Gerhards CC (2007) Effect of moisture content and temperature on the mechanical properties of wood: an analysis of immediate effects. Wood Fiber Sci 14:4-36

Gindl W, Sretenovic A, Vincenti A, Muller U (2005) Direct measurement of strain distribution along a wood bond line. Part 2: effects of adhesive penetration on strain distribution. Holzforschung 59:307-310

Grøstad K, Pedersen A (2010) Emulsion polymer isocyanates as wood adhesive: a review. J Adhes Sci Technol 24:1357-1381. https://doi.org/10.1163/016942410x500981

Hassani MM (2015) Adhesive bonding of structural hardwood elements. Dissertation, ETH Zurich 
Henriques A, Coelho C, Ferra JM, Martins JM, Magalhaes FD, Carvalho L (2017) Wood composites: materials, manufacturing and engineering, vol 6. Walter de Gruyter GmbH \& Co KG, Munich

Jakes JE, Hunt CG, Yelle DJ et al (2015) Synchrotron-based X-ray fluorescence microscopy in conjunction with nanoindentation to study molecular-scale interactions of phenol-formaldehyde in wood cell walls. ACS Appl Mater Interfaces 7:6584-6589

Keylwerth R, Noack D (1964) Chamber drying of sawn wood. Eur J Wood Prod 22:29-36

Kläusler O, Clauß S, Lübke L, Trachsel J, Niemz P (2013) Influence of moisture on stress-strain behaviour of adhesives used for structural bonding of wood. Int J Adhes Adhes 44:57-65. https://doi. org/10.1016/j.ijadhadh.2013.01.015

Kollmann F (1982) Technology of wood and wood-based materials, vol 1-2. Springer, Berlin

Konnerth J, Gindl W (2008) Observation of the influence of temperature on the mechanical properties of wood adhesives by nanoindentation. Holzforschung 62:714-717. https://doi.org/10.1515/ hf. 2008.108

Konnerth J, Gindl W, Müller U (2006) Elastic properties of adhesive polymers. I. Polymer films by means of electronic speckle pattern interferometry. J Appl Polym Sci 103:3936-3939. https://doi. org/10.1002/app.24434

Mannes D, Schmidt JA, Niemz P, Volkmer T (2012) Investigation on the influence of the adhesive type on capillary water transport in wood parallel to the grain direction. Bauphysik 34:61-65

Myers GE (1984) How mole ratio of UF resin affects formaldehyde emission and other properties: a literature critique. For Prod J 34:35-41

Niemz P, Sonderegger W (2017) Wood physics: physics of wood and wood-based materials. Carl Hanser Verlag GmbH Co KG, Munich

Qiao L, Easteal AJ, Bolt CJ, Coveny PK, Franich RA (2000) Thermomechanical analysis and performance tests of some EPI wood adhesives. Pigment Resin Technol 29:229-237

Rapp AO, Sudhoff B, Pittich D (2011) Damage to wooden floors, vol 2. Fraunhofer-IRB-Verlag, Stuttgart

Rindler A, Vay O, Hansmann C, Müller U (2017) Dimensional stability of multi-layered wood based panels: a review. Wood Sci Technol 51:969-996. https://doi.org/10.1007/s00226-017-0940-7

Rindler A, Pöll C, Hansmann C, Müller U, Konnerth J (2018) Moisture related elastic and viscoelastic behaviour of wood adhesives by means of in situ nanoindentation. Int J Adhes Adhes 85:123-129. https://doi.org/10.1016/j.ijadhadh.2018.06.004

Šebenik U, Krajnc M (2007) Influence of the soft segment length and content on the synthesis and properties of isocyanate-terminated urethane prepolymers. Int J Adhes Adhes 27:527-535

Stöckel F, Konnerth J, Kantner W, Moser J, Gindl W (2010) Tensile shear strength of UF- and MUFbonded veneer related to data of adhesives and cell walls measured by nanoindentation. Holzforschung 64:56. https://doi.org/10.1515/hf.2010.046

Stöckel F, Konnerth J, Gindl-Altmutter W (2013) Mechanical properties of adhesives for bonding wood: a review. Int J Adhes Adhes 45:32-41. https://doi.org/10.1016/j.ijadhadh.2013.03.013

Suchsland O, McNatt JD (1986) Computer simulation of laminated wood panel warping. For Prod J 36:16-23

Tobisch S (2006) Methods to influence the selected properties of three-layer solid wood panels made of softwood. Dissertation, Universität Hamburg

Vailati C, Rüggeberg M, Burgert I, Hass P (2018) The kinetics of wooden bilayers is not affected by different wood adhesive systems. Wood Sci Technol 52:1589-1606

Wagenführ A, Scholz F (2012) Paperback of wood technology, vol 1. Carl Hanser Verlag GmbH Co KG, Munich

Wimmer R, Kläusler O, Niemz P (2013) Water sorption mechanisms of commercial wood adhesive films. Wood Sci Technol 47:763-775. https://doi.org/10.1007/s00226-013-0538-7

Publisher's Note Springer Nature remains neutral with regard to jurisdictional claims in published maps and institutional affiliations. 